\title{
Crystal structure and electronic properties of new compound $\mathrm{Zr}_{6.5} \mathrm{Pt}_{6} \mathrm{Se}_{19}$
}

Hanlin Wu ${ }^{\dagger}$ Huifei Zhai, ${ }^{\dagger}$ Sheng Li, ${ }^{\dagger}$ Maurice Sorolla II, ${ }^{\S}$ Gregory T. McCandless, ${ }^{\ddagger}$ Daniel Peirano Petit, ${ }^{\dagger}$ Julia Y. Chan, $\stackrel{\dagger}{\dagger}$ and Bing Lv*,†

†Department of Physics, The University of Texas at Dallas, Richardson, Texas 75080, United States

$\S$ Department of Chemistry, University of Houston, Houston, Texas 77204, United States

Department of Chemistry and Biochemistry, The University of Texas at Dallas, Richardson, Texas 75080, United States

\section{Contents:}

1. Table S1: Selected interatomic angles for $\mathrm{Zr}_{6.5} \mathrm{Pt}_{6} \mathrm{Se}_{19}$.

2. Figure S1: Integrated precession images of different planes for $\mathrm{Zr}_{6.5} \mathrm{Pt}_{6} \mathrm{Se}_{19}$.

3. Figure S2: COHP plot of the nearest-neighboring $\mathrm{Zr}-\mathrm{Se}$ interactions in $\mathrm{Zr}_{6.5} \mathrm{Pt}_{6} \mathrm{Se}_{19}$ model calculated using TB-LMTO-ASA.

4. Figure S3: COHP plot of the nearest-neighboring Pt-Se interactions in $\mathrm{Zr}_{6.5} \mathrm{Pt}_{6} \mathrm{Se}_{19}$ model calculated using TB-LMTO-ASA.

5. Figure S4: COHP plot of the nearest-neighboring Zr-Pt interactions in $\mathrm{Zr}_{6.5} \mathrm{Pt}_{6} \mathrm{Se}_{19}$ model calculated using TB-LMTO-ASA. 
Table S1. Selected Interatomic Angles for $\mathrm{Zr}_{6.5} \mathrm{Pt}_{6} \mathrm{Se}_{19}$.

\begin{tabular}{|c|c|c|c|c|c|}
\hline \multicolumn{3}{|c|}{ Angle (deg) } & \multicolumn{3}{|c|}{ Angle (deg) } \\
\hline $\mathrm{Se} 5-\mathrm{Zr} 1-\mathrm{Se} 5$ & $(\times 1)$ & $88.48(7)$ & $\mathrm{Se} 2-\mathrm{Pt} 1-\mathrm{Se} 2$ & $(\times 1)$ & $94.79(4)$ \\
\hline $\mathrm{Se} 5-\mathrm{Zr} 1-\mathrm{Se} 6$ & $(\times 2)$ & $177.32(6)$ & $\mathrm{Se} 1-\mathrm{Pt} 1-\mathrm{Se} 2$ & $(\times 2)$ & $170.15(3)$ \\
\hline $\mathrm{Se} 5-\mathrm{Zr} 1-\mathrm{Se} 6$ & $(\times 2)$ & $94.19(3)$ & $\mathrm{Se} 2-\mathrm{Pt} 1-\mathrm{Se} 1$ & $(\times 2)$ & $84.56(3)$ \\
\hline $\mathrm{Se} 6-\mathrm{Zr} 1-\mathrm{Se} 6$ & $(\times 1)$ & $83.13(6)$ & $\mathrm{Se} 1-\mathrm{Pt} 1-\mathrm{Se} 1$ & $(\times 1)$ & 94.39(4) \\
\hline $\mathrm{Se} 5-\mathrm{Zr} 1-\mathrm{Se} 1$ & $(\times 4)$ & $94.29(3)$ & $\mathrm{Se} 2-\mathrm{Pt} 1-\mathrm{Se} 5$ & $(\times 2)$ & $89.58(3)$ \\
\hline Se6-Zr1-Se1 & $(\times 4)$ & $85.52(3)$ & $\mathrm{Se} 1-\mathrm{Pt} 1-\mathrm{Se} 5$ & $(\times 2)$ & $100.24(3)$ \\
\hline \multirow[t]{2}{*}{$\mathrm{Se} 1-\mathrm{Zr} 1-\mathrm{Se} 1$} & $(\times 1)$ & $168.02(8)$ & & & \\
\hline & & & $\mathrm{Se} 3-\mathrm{Pt} 2-\mathrm{Se} 3$ & $(\times 1)$ & $91.71(5)$ \\
\hline $\mathrm{Se} 2-\mathrm{Zr} 2-\mathrm{Se} 3$ & $(\times 2)$ & $133.68(2)$ & $\mathrm{Se} 3-\mathrm{Pt} 2-\mathrm{Se} 4$ & $(\times 2)$ & $88.25(4)$ \\
\hline $\mathrm{Se} 3-\mathrm{Zr} 2-\mathrm{Se} 3$ & $(\times 1)$ & $85.01(4)$ & $\mathrm{Se} 3-\mathrm{Pt} 2-\mathrm{Se} 4$ & $(\times 2)$ & $179.96(3)$ \\
\hline $\mathrm{Se} 2-\mathrm{Zr} 2-\mathrm{Se} 4$ & $(\times 2)$ & $84.86(3)$ & $\mathrm{Se} 4-\mathrm{Pt} 2-\mathrm{Se} 4$ & $(\times 1)$ & $91.80(5)$ \\
\hline $\mathrm{Se} 3-\mathrm{Zr} 2-\mathrm{Se} 4$ & $(\times 2)$ & $134.90(4)$ & & & \\
\hline $\mathrm{Se} 3-\mathrm{Zr} 2-\mathrm{Se} 4$ & $(\times 2)$ & $78.25(3)$ & & & \\
\hline $\mathrm{Se} 4-\mathrm{Zr} 2-\mathrm{Se} 4$ & $(\times 1)$ & $84.60(4)$ & & & \\
\hline $\mathrm{Se} 2-\mathrm{Zr} 2-\mathrm{Se} 1$ & $(\times 1)$ & $75.76(4)$ & & & \\
\hline $\mathrm{Se} 3-\mathrm{Zr} 2-\mathrm{Se} 1$ & $(\times 2)$ & $85.41(3)$ & & & \\
\hline $\mathrm{Se} 4-\mathrm{Zr} 2-\mathrm{Se} 1$ & $(\times 2)$ & $133.60(2)$ & & & \\
\hline
\end{tabular}



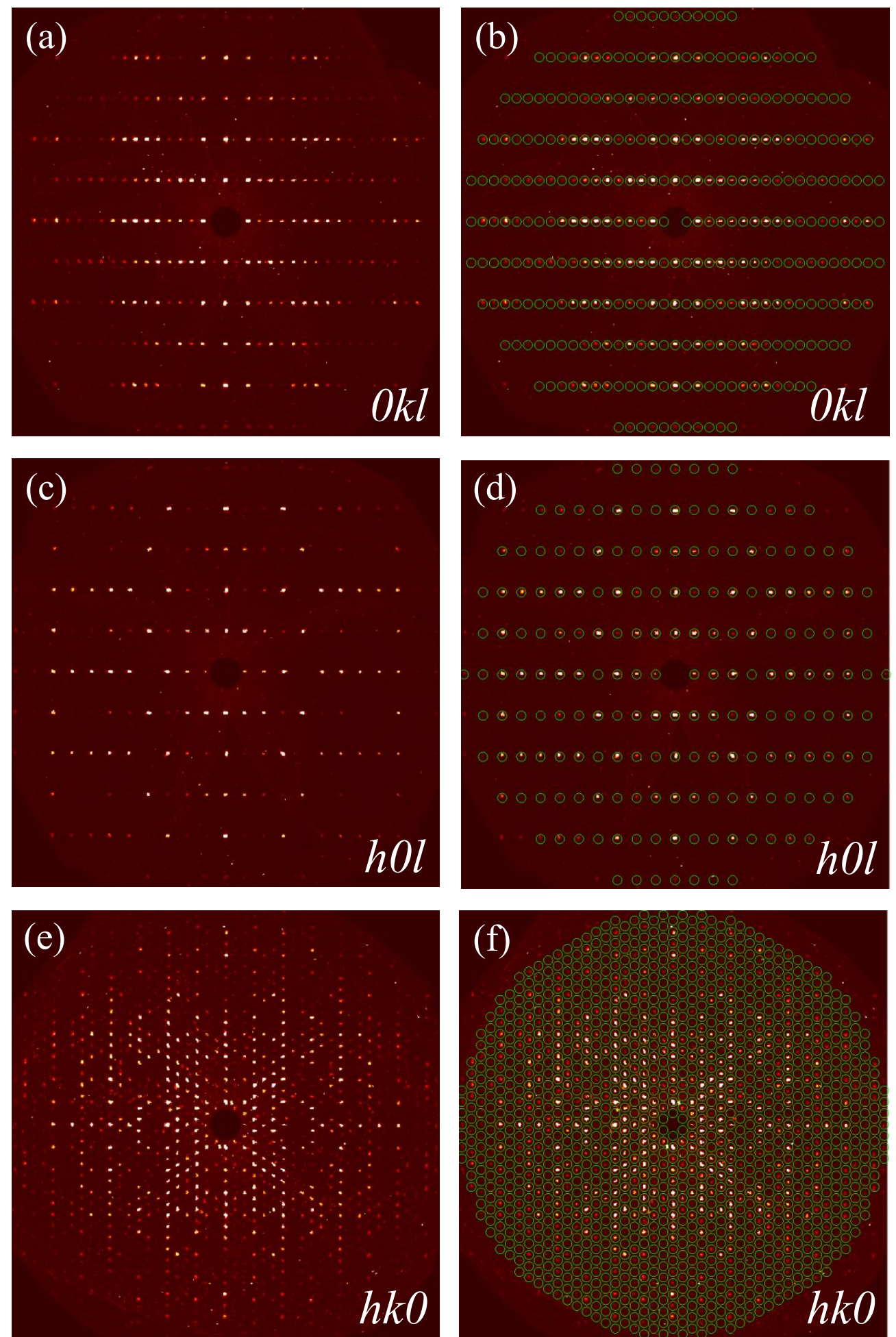

Figure. S1 Integrated precession images of (a) $0 k l$, (c) $h 0 l$ and (e) $h k 0$ layer with all detected reflections are indexed as circled spots in (b), (d) and (f), respectively. 
(a)
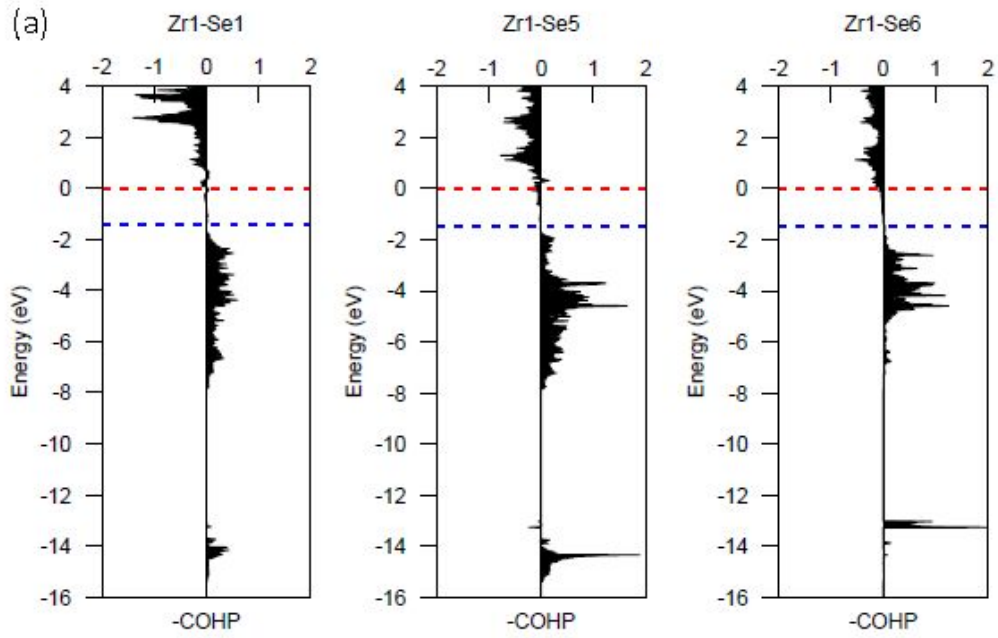

(b)
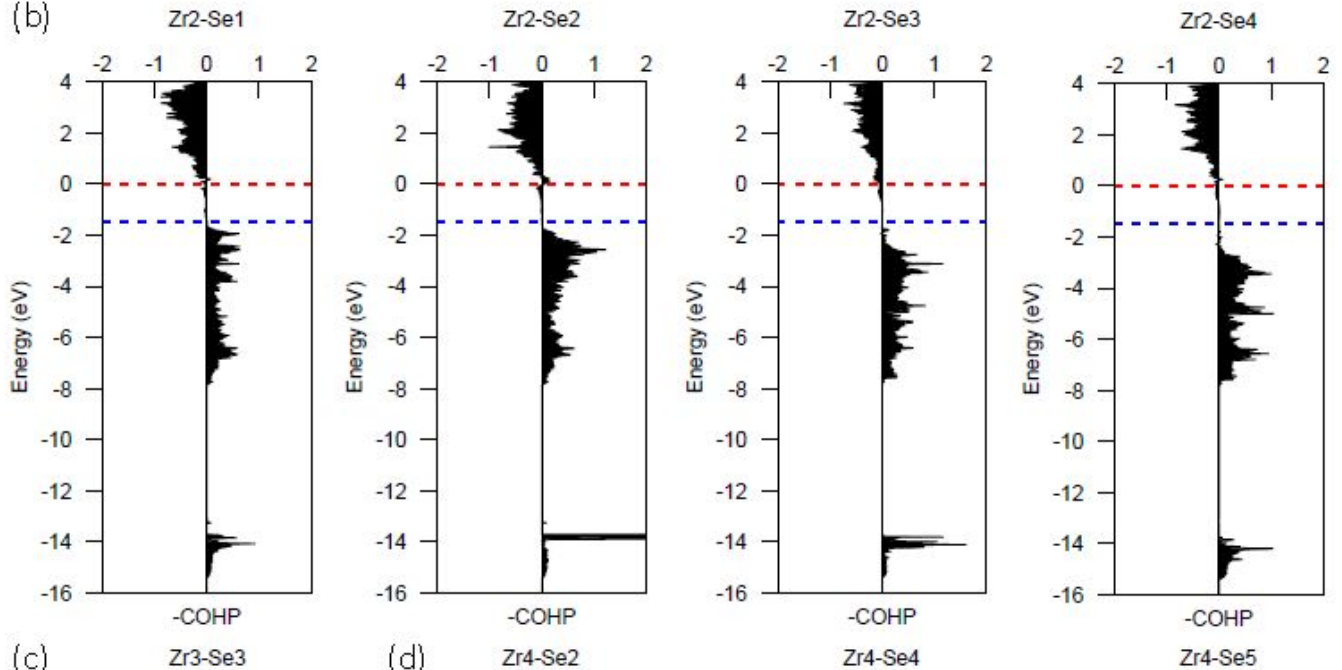

(c)

(d)
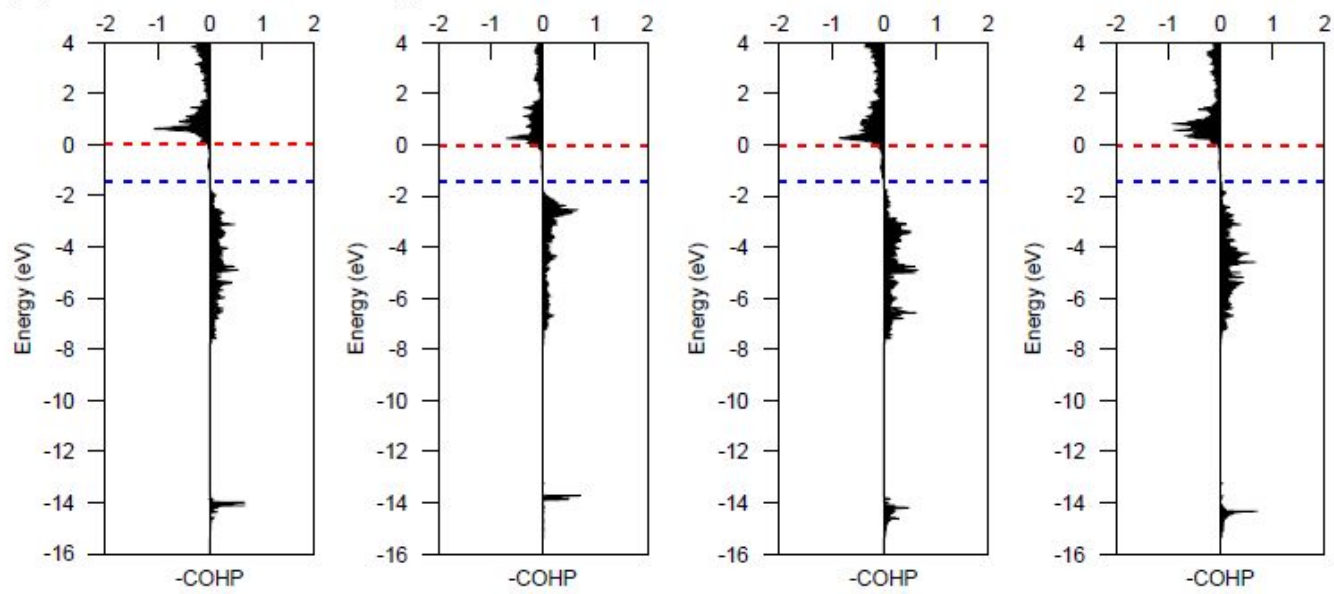

Figure S2. Crystal Orbital Hamilton Population (-COHP) of the nearest-neighboring (a) Zr1$\mathrm{Se}$,(b) $\mathrm{Zr2}$-Se, (c) $\mathrm{Zr} 3-\mathrm{Se}$ and (d) $\mathrm{Zr} 4-\mathrm{Se}$ interactions in $\mathrm{Zr}_{6.5} \mathrm{Pt}_{6} \mathrm{Se}_{19}$ model calculated using TBLMTO-ASA. Red dash line denote all $\mathrm{Zr}$ sites are fully occupied with total electron count of $210 e^{-}$. Blue dashed line denote partially occupied $\mathrm{Zr}$ sites for $\mathrm{Zr}_{6.5} \mathrm{Pt}_{6} \mathrm{Se}_{19}$ compound with total electron count of $200 e^{-}$. Fermi Level is set at $0 \mathrm{eV}$ 
(a)

Pt1-Se1

Pt1-Se2

Pt1-Se5
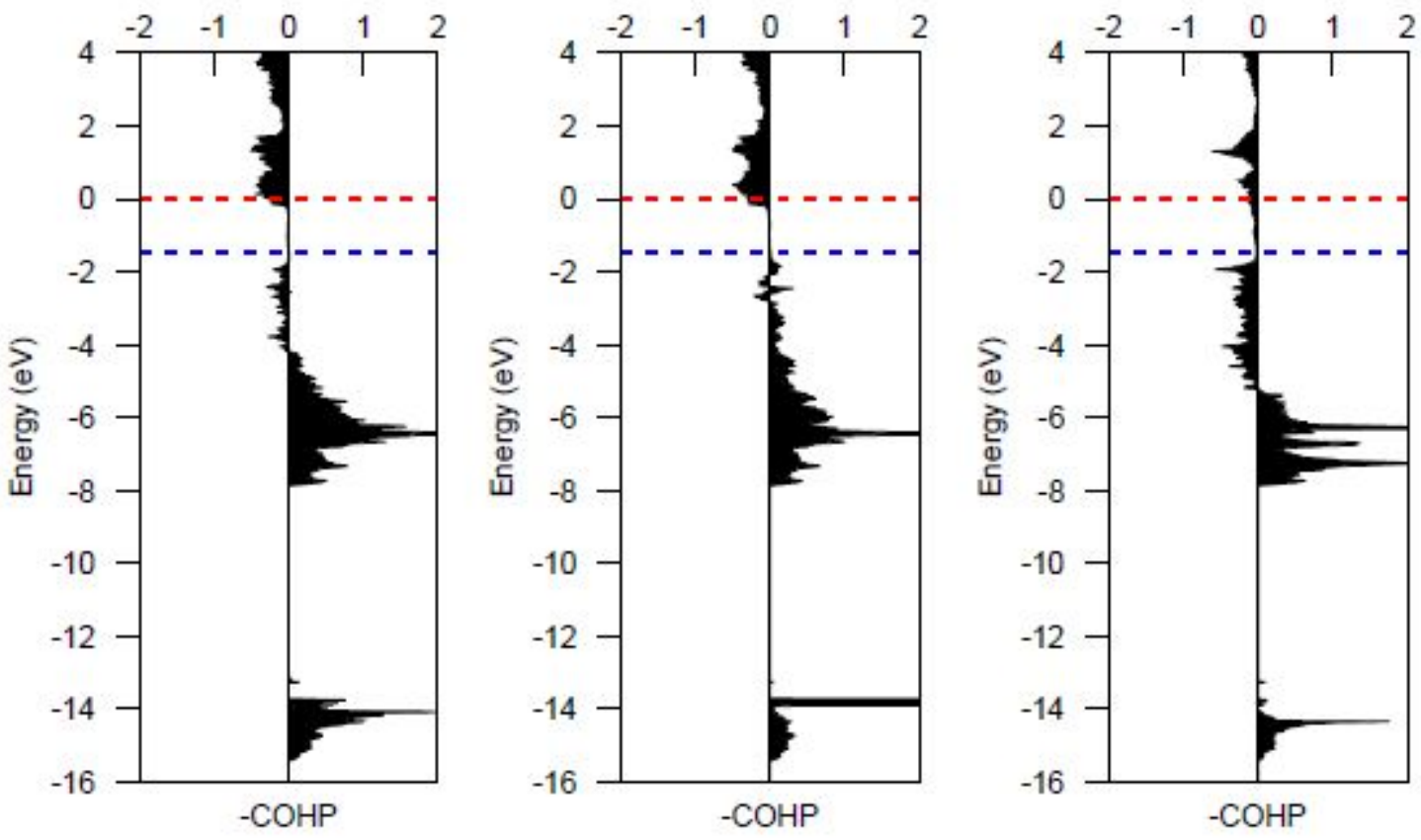

(b)

Pt2-Se3
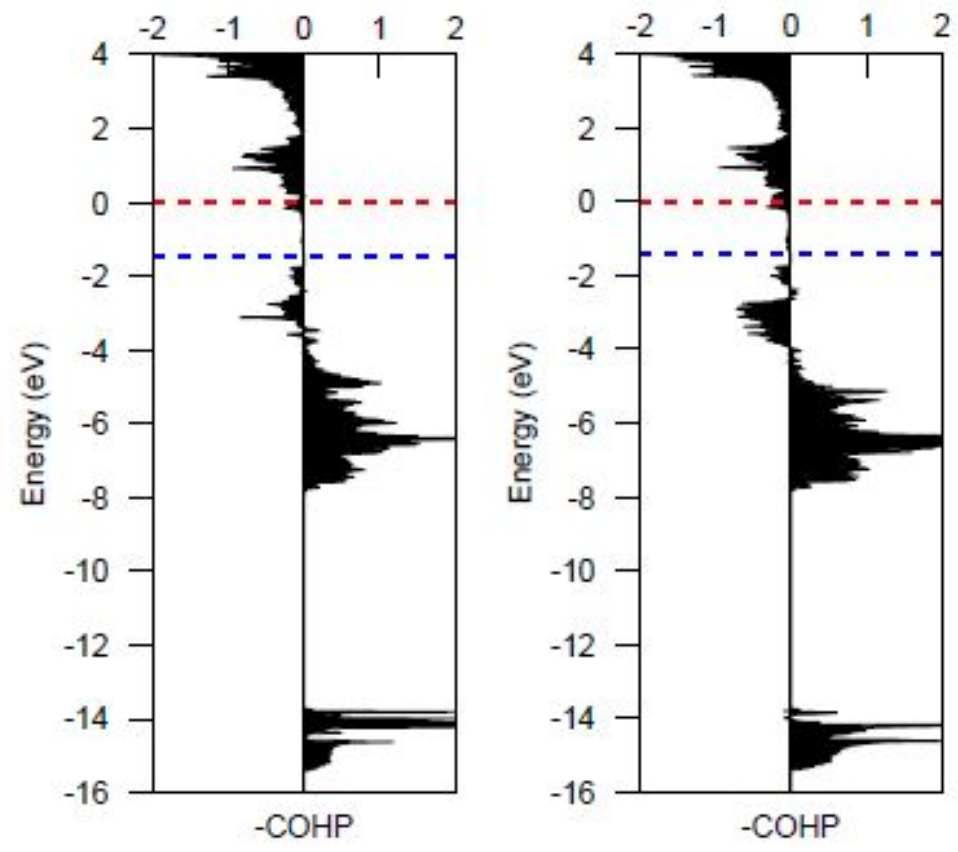

Figure S3. Crystal Orbital Hamilton Population (-COHP) of the nearest-neighboring (a) Pt1-Se and (b) Pt2-Se interactions in $\mathrm{Zr}_{6.5} \mathrm{Pt}_{6} \mathrm{Se}_{19}$ model calculated using TB-LMTO-ASA. Red dash line denote all $\mathrm{Zr}$ sites are fully occupied with total electron count of $210 e^{-}$. Blue dashed line denote partially occupied $\mathrm{Zr}$ sites for $\mathrm{Zr}_{6.5} \mathrm{Pt}_{6} \mathrm{Se}_{19}$ compound with total electron count of $200 e^{-}$.. Fermi Level is set at $0 \mathrm{eV}$ 


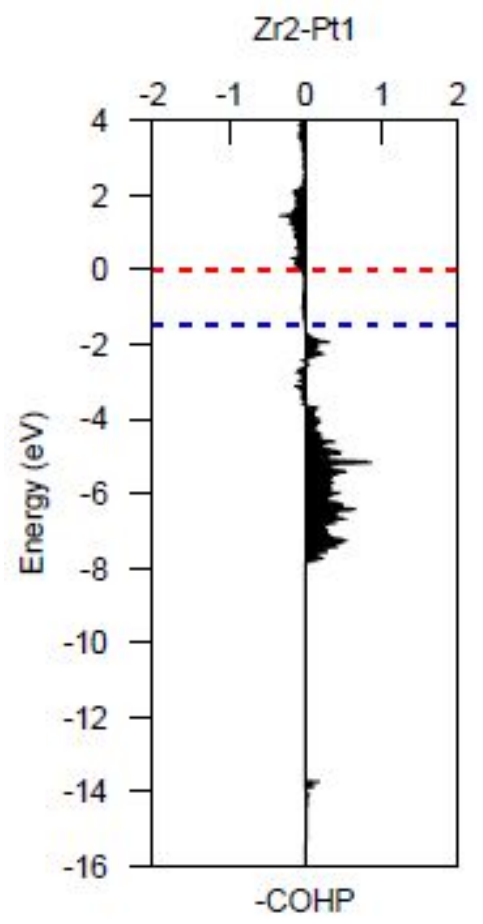

Figure S4. Crystal Orbital Hamilton Population (-COHP) of the nearest-neighboring Zr-Pt interactions in $\mathrm{Zr}_{6.5} \mathrm{Pt}_{6} \mathrm{Se}_{19}$ model calculated using TB-LMTO-ASA. Red dash line denote all $\mathrm{Zr}$ sites are fully occupied with total electron count of $210 e^{-}$. Blue dashed line denote partially occupied $\mathrm{Zr}$ sites for $\mathrm{Zr}_{6.5} \mathrm{Pt}_{6} \mathrm{Se}_{19}$ compound with total electron count of $200 e^{-}$. Fermi Level is set at $0 \mathrm{eV}$ 